the patient on his side. A double hip spica is applied with a window anteriorly large enough to accommodate the Hamilton Irving box.

The urethra may also be ruptured in association with a fracture-dislocation of the pelvis. An attempt should be made to reduce the dislocation at once under anaesthesia with the patient on his side. If this is successful, a catheter should be passed. If, however, reduction of the fracturedislocation is not effected, or, if the catheter will not pass following reduction an immediate suprapubic cystostomy is indicated. The writer has recently seen a fracture-dislocation of the pelvis with a damaged urethra in which the fatal black spot on the glans penis, due to gangrene, pronounced the fact that a supra-pubic cystostomy had been left until too late.

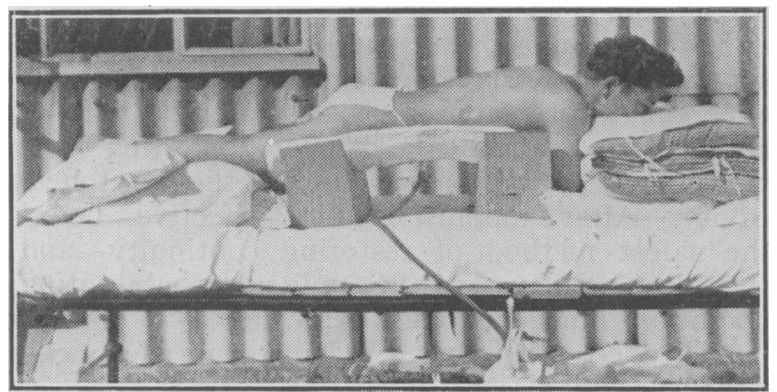

Author's method of treatment of fracture-dislocation of the spine, associated with paraplegia (see Text).

(Reproduced by kind permission of the "Medical Press and Circular.')

\section{CLOSURE OF COLOSTOMIES}

\section{By Major D. Lang Stevenson, F.R.C.S.}

Success in closure of colostomy and faecal fistula depends on a thorough investigation and preparation of the patient, with attention to every detail in operative technique and in post-operative treatment. The following article deals briefly with some general principles and describes a method of extraperitoneal closure of artificial openings in the bowel, which has given most satisfactory results.

\section{Preparation of the Patient}

In colon surgery it is important to bear in mind the different contents and function of the right and left tracts. As the right colon contains easily propelled fluid and is not subjected to the same tensions and trauma as the left, it can be treated surgically like small bowel. However, it would be disastrous to deal with the unprepared left colon in the same way, as faecal masses, gaseous distension, and the relatively poor blood supply of the bowel wall greatly endanger the suture line. It is therefore a first importance that the left colon is most carefully cleansed for a planned operation. This is achieved by a tap-water washout of the distal colon; in the morning from the colostomy and in the evening from the rectum. In some cases it may be a week or more before the returning fluid is clear. Scybalous masses can be softened by a slow continuous drip of warm water given by a catheter into the colostomy. If there is any tendency for the colostomy to be constipated, which is unusual, senna tea is given and the diet is suitably "modified. The day before operation a liquid non-residue diet is commenced and, for the first time, the proximal bowel is washed out with tap water. Two hours before operation both limbs of the colostomy are given a local cleansing with a half-pint of soapy water, all of which must be aspirated. The colostomy stoma is then irrigated with hydrogen peroxide followed by saline, and finally a plug of gauze soaked in flavine $\mathrm{I} / \mathrm{IOOO}$ is tucked into the opening. After the skin has been thoroughly washed with soap and water for five minutes it is painted with flavine and spirit. On the theatre table the skin receives a further application of the same antiseptic.

- When constitutional effects are evident, general treatment in the form of a high protein and a high vitamin diet and fresh blood transfusions over a period of weeks, are invaluable. A course of Succinyl Sulphathiazole before an intraperitoneal operation, or where wound infection is perpetuated by a faecal fistula, is worth while in reducing the virulence of the bacterial flora. Four tablets five-times-a-day should be given three days before and three days after operation.

Any discharging sinuses at or near the site of a previous faecal fistula may be investigated by opaque media, and, if necessary, surgically explored; but on no account should an abdominal sinus of this nature be curetted or forcibly probed.

\section{Operation}

In the straightforward case the wounds are healed, a well-formed loop colostomy with no loss of bowel wall is present, and the distal gut is intact. Here, contrary to frequently expressed opinion, extraperitoneal repair gives uniformly good results if a sufficiently extensive mobilisation of the loop is carried out. This highly important part of the operation straightens out the temporary angulation or apparent spur in the bowel, allows the suture to be completed without tension, and restores the bowel to an unrestricted and good functional position. The word extraperitoneal is used in the sense that the loop of bowel is freed 
without opening: the peritoneal cavity. The limiting membrane may be, and often is, a layer of adventitious adhesions which provide adequate isolation.

An elliptical incision is made a quarter-inch from the stoma and extended about one-inch above and below it. As a rule the incision can be made cleanly through subcutaneous tissue, but in some instances the gut may be just under the skin. Care must therefore be taken to undercut cautiously if this is suspected. To repeat, extraperitoneal mobilisation is the essential stage in the operation. The secret in this step; as in all surgical dissection, is the correct application of tension on the appropriate tissues. To obtain the maximum advantage from this principle, with the minimum injury to the friable edge of the colostomy, a series of cotton stay sutures are passed at intervals through the strip of adherent skin around the entire circumference of the opening. Six to ten of these stays are used-according to the size of the stoma. The cotton stays are gathered together, put on slight tension by a straight upward pull, and then twisted so that the whole fasciculas can be grasped with a strong pair of artery forceps. Using the forceps as a tractor even tension can be exerted on the whole loop or any desired segment of it, without damage to the bowel wall-a point of considerable importance in ensuing dissection. With two pairs of strong tissue forceps the assistant raises and draws each layer of the abdominal wall in turn away from the colostomy, while the surgeon applies gentle traction on the loop in the opposite direction. The dissection proceeds circumferentially by light touches of a sharp knife. The right plain is easily visualised in this way and adequate mobilisation can be carried out without danger of opening the bowel or peritoneal cavity. Once the muscular layers of the abdominal wall have been separated off, scissors are more useful for dividing the remaining strands of anchoring tissue. The smallest bleeding point must be ligatured with fine catgut throughout the operation.

The loop is then isolated carefully with packs and the stay sutures removed. The adherent skin is neatly trimmed away with scissors, but no sacrifice of the mucosa must be made. Meticulous care should be taken to regain the original length of the rolled everted colostomy margin by gentle dissection. If this is done and careful suture is carried out, kinking and bulky infolding which results in breakdown or late subacute obstruction is avoided. The flavine plug is then removed with forceps and both are discarded. It is undesirable, and usually unnecessary, to put a finger into the colostomy during the operation. When the loop has been correctly orientated two stay sutures are placed at the extreme angle of the transverse axis of the opening, and tension on these approximates the edges for suture. Repair is carried out in two layers, the mucosa by a con- $\frac{\mathbb{\Phi}}{\mathbb{Q}}$ tinuous catgut 000000 , and the seromuscular by $\stackrel{\circ}{\llcorner}$ Halsted sutures of fine silk placed closely and $\stackrel{\Rightarrow}{\Rightarrow}$ tied lightly. Patency of the lumen should always be obvious to the examining fingers. The suture line is cleansed with flavine solution and the $\frac{\bar{O}}{\mathrm{O}}$ towels removed. Fresh gloves and instruments $\frac{\bar{p}}{\frac{\bar{\sigma}}{\sigma}}$ are provided for the repair of the abdominal $\underset{\Omega}{\Omega}$ wound which is insufflated with Sulphathiazole powder.

Only if destruction of the bowel substance or $\vec{\circ}$ distortion of local anatomy makes an extra-peri- $\vec{\overrightarrow{ }}$ toneal repair impracticable need an intra- ab- $\omega$ dominal operation be resorted to. In such a case the preliminary procedure is the same. The 3 . peritoneum is then opened at a safe point, where it is usually almost transparent, and a finger is $\vec{\sim}$ slipped around the colostomy component. The $f$ adherent peritoneum is thus put on a stretch and divided over the finger, allowing the whole loop to be drawn out of the peritonealo cavity. After mobilising the defective bowelthe safest method of restoring continuity-ando this last and relatively minor rehabilitating operation must be safe-is to form a double-barrelled colostomy with a long spur which cann later be easily closed.

It might be mentioned at this point that althoug cr closure of this type of deliberately forme colostomy is a well-established, simple and safe procedure, the enterotribe may be used inadvisedly, particularly if the opening is in the mid line. Unless records of the previous operation are avail- $\frac{\mathrm{Q}}{\mathrm{Q}}$

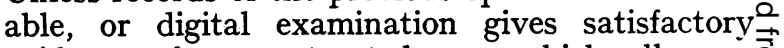
evidence of a constructed spur which allows a을 finger in either loop to be approximated, it is better to obtain further information by surgical mobilisation of the loop. The Oschner de Bakey instrument, or one of its modifications, is the mosto satisfactory enterotribe. About eight days are necessary for the crushing, and the final closure 3 . should not be done until the resulting oedema hasi settled down. Seton Pringle's method of sur-굴 rounding the duplicated bowel with vaseline gauze (silver leaf is a non-greasy alternative) to prevent it becoming adherent to the abdominalo wall, is an advantage in effecting a spontaneous or very simple closure after crushing of the spur. $N$

Should circumstances prevail which make an end to-end anastomosis or plastic repair, the method of choice, this should be carried out formally with allw the precautions available for safe colon surgery. The bowel ends are resected, and this is done obliquely $\subseteq$ A slender pair of special intestinal forceps (maded by Genito-Urinary Mfg. Coy. under the writer's name) greatly facilitates anastomosis. The safety 
of the colonic suture may be further ensured by so mobilising two leaves of the parietal peritoneum and stitching them with an atraumatic needle to the repaired segment of the bowel and mesentery, that this part lies in an extraperitoneal compartment. This protective isolation can be carried out neatly without altering the normal course of the bowel if the colostomy has been situated in the flank.

\section{Repair of the Abdominal Wall}

While the first aim of the operation is healing of the repaired bowel, prevention of an infected wound is of next importance. A strong abdominal wall and a quick convalescence is most likely to be achieved by avoiding. collections of incarcerated serum or blood, which are the usual causes of this complication. The following method of sewing up the abdominal wall has proved satisfactory.

The layers of the abdominal wall are first identified and cleaned. The transverse muscle or first abdominal layer is closed with interrupted sutures of No. I plain catgut, allowing the repaired bowel to lie comfortably in the extraperitoneal space. Deep silkworm sutures, left untied, are passed through all layers including the skin, and a flavine pack is placed lightly in the wound. In forty-eight hours the pack is removed and the sutures tied. Where the wound is a large one, or if it is in the mid line, it is better to do a formal secondary suture on the third day, using interrupted sutures only. If there is any evidence of infection when the pack is removed irrigations are carried out with hydrogen peroxide and saline four-hourly until the wound is clean. Secondary suture is then done.

\section{Post-operative Treatment}

Watery liquids only are given for five days and a warm catheter-more comfortable than a flatus tube-is left in the rectum at intervals for the greater part of each day. Liquid paraffin, one tablespoonful morning and evening, and an infusion of six pods of senna is commenced on the fifth day. The latter is by far the most satisfactory non-griping laxative that can be used in a surgical ward. The former is valuable in reducing any mild trauma caused by the passage of faeces, and there should be no danger of seepage if the suture line is sound. On the same day small, semi-solid three-hourly feeds are commenced and on the seventh day a warm olive oil enema (6 ozs.) is given. An hour or so later, or when the patient feels inclined, he is allowed to get up and use a commode, when, as a rule, he has a normal bowel action. Sutures are removed on the tenth day.

Small feeds should gradually be increased during the next four weeks and the motions kept soft by diet and the laxative mentioned.

\section{A HANDBOOK ON DISEASES OF CHILDREN 4th edition}

By Bruce Williamson. Published by E. \& S. Livingstone Ltd., Edinburgh. 1945. Price r2s. 6d.

Painfully conscious of the truth of the Greek aphorism that a big book is a big evil, many students and practitioners of medicine will be grateful for a new edition of this popular $H a n d b o o k$, of which the last edition, published in r942, had been out of print for over a year. For its size ( $7 \frac{1}{2}$ ins. $\times 5$ ins., 388 pages), printing and binding, are attractive; it is concise, modern, reliable, and easy to read; and, without apparent effort, it succeeds in instilling common sense and wisdom into the reader's mind. The 81 illustrations are for the most part excellent, though the coloured plate showing the rash in scarlatina is disappointing.

In addition to detailed discussions of the use of the sulphanilamides in the pneumonias and in meningococcal meningitis, there is a special section on sulphanilamide therapy and a note on penicillin. The chapter on the vitamins which introduces the problem of war-nutrition is up to date, and the author has many shrewd and illuminating things to say on the functional nervous disorders.

It is suggested that in the next edition the following sections be enlarged: " $D$ and $V$ " in infants; "tonsils and adenoids" (quinsy is not mentioned); and the diagnostic tuberculin tests. John Thomson taught that Fowler's solution is a specific in lienteric diarrhoea, and the reviewer in his student days was impressed with the danger of whooping cough fertilising the soil for the tubercle bacillus. Has the teaching in these respects changed?

Ramstedt, not Rammstedt, is the correct spelling, and "Tay-Sach's" should read "Tay-Sachs'."

\section{DISEASES OF THE NERVOUS SYSTEM 4th edition}

By F. M. R. Walshe. E. \& S. Livingstone Ltd., Edinburgh. 1945. Price I5s.

The popularity of this book is shown by the appearance of yet another edition. It has in addition been reprinted twice since it was first published in September, 1940.

It sets out, most successfully, to present a simple outline of the subject and is particularly well balanced in its presentation of new material. The new edition is 12 pages longer than its predecessor, which is perhaps a pity, as one of the great features of the book has been its brevity, but even so there are only 350 pages of good-sized print.

There are some errors in the indexing, e.g. Dystrophy Muscular, p. 222 for p. 226, and Herpes Zoster, p. I 53 for p. 156. There are also a larger number of errors in the references to the Figs. given in the text, e.g. on p. 239 Fig. 33 for Fig. 36, on p. 227 Fig. 30D for Fig. 33D, on p. 228 Fig. 31 for Fig. 34, on p. 216 Fig. 28 for Fig. 3I, etc. Though of more importance in a reference book, such minor errors are always annoying to the reader.

Some may doubt if the Wassermann Reaction can ever properly be described as unnecessary, particularly those who have seen the tragedy of a diagnosis missed in the early stages of Neurosyphilis before the clinical diagnosis has become obvious and the structural damage irreversible. Whatever views may be correct regarding the frequency of Neurosyphilis in different periods of peace and war, it remains one of the most important therapeutic fields in Neurology.

The paper has suffered surprisingly little from two more years of war, and Messrs. Livingstone may be congratulated on keeping the price the same, even including five additional illustrations. 